Running Title: BINDING IN PIGEONS

\title{
Evidence for Object-Place binding in pigeons in a sequence-learning procedure
}

\author{
Aaron P. Blaisdell \& Julia E. Schroeder \\ Department of Psychology, University of California, Los Angeles, CA, USA
}

Correspondence:

Aaron Blaisdell

UCLA Department of Psychology

1285 Franz Hall

Los Angeles, CA 90095-1563, USA

blaisdell@psych.ucla.edu 


\begin{abstract}
We studied object-location binding in pigeons using a sequence learning procedure. A sequence of four objects was presented, one at a time at one of four locations on a touchscreen. A single peck at the object ended the trial, and food reinforcement was delivered intermittently. In Experiment 1, a between-subjects design was used to present objects, locations, or both in a regular sequence or randomly. Response time costs on nonreinforced probe tests on which object order, location order, or both were disrupted revealed sequence learning effects. Pigeons encoded location order when it was consistent, but not object order when it alone was consistent. When both were consistent, pigeons encoded both, and also showed evidence of object-location binding. In Experiment 2, two groups of pigeons received training on sequences where the same object always appeared at the same location. For some pigeons a consistent sequence was used while for others sequence order was randomized. Only when sequence order was consistent was object-location binding found. These experiments are the first demonstrations of strong and lasting feature binding in pigeons.
\end{abstract}

Keywords: pigeons, biding, sequence learning 
When we open our eyes, we see a world populated by objects. Objects appear as coherent sets of features and properties that become bound together in perception. There's been a prodigious amount of work investigating perceptual binding since the seminal work by Anne Treisman and her associates (Treisman \& Gelade, 1980). One emergent finding from this literature is that attention is a necessary driver of binding. Experiments performed in monkeys (Luck et al., 1997) and humans (Kastner et al., 1998) have identified common neural mechanisms of attention that modulate perceptual binding. Moreover, attention appears to play an important role in the storage of feature-biding in memory, what we will refer to as "memory feature biding" to contrast it to "perceptual feature binding". We believe both perceptual and memory feature binding involve the same perceptual binding mechanisms, but are shown under different conditions and using different procedures. Specifically, perceptual feature binding is demonstrated in tasks that show an immediate effect during stimulus presentation and encoding. Memory feature binding, on the other hand, is typically shown on a memory test for prior learning, or after a lot of practice, such as on a Serial Reaction Time (SRT) task. Given the similar role of attentional processes in binding in both monkeys and humans, this raises the question of how broadly across the animal kingdom does attention modulate binding? Does binding in other species require attentional processes? We investigated this question by studying the role of attention in binding in pigeons.

Pigeons are an ideal animal model in which to study this question. There is much known about the learning, memory, and perceptual capacities of pigeons, which serve as one of the most common non-mammalian models in psychology (Cook, 2001). The avian visual system has evolved functional homologies to the primate visual system in the ability to rapidly analyze visual information (e.g., Cook, 2000; Husband \& Shimizu, 2001; Wylie et al., 2009). Furthermore, pigeons show many of the same attentional (Blough, 1991; Blough \& Blough, 1997; Cook, Katz, \& Blaisdell, 2012) and perceptual (Cook, 1992a, 1992b) processes necessary to support binding. Despite these homologies, the few studies that have directly looked for perceptual binding in pigeons have found weak or no evidence (Katz, Cook, \& Magnotti, 2010; Lazareva \& Wasserman, 2016). Lazareva and Wasserman used a change-detection task in which, on each trial, pigeons were presented with two consecutive multi-item displays. Pigeons were reinforced for pecking one key if the displays were identical and another key if the items in the displays changed in color, orientation, and location. After learning this discrimination, feature binding was tested by swapping one (Experiment 1) or two (Experiment 2) features between 
items in the display. For example, if the first display contained a red horizontal bar in the upper quadrant and yellow vertical bar in the lower quadrant, then the second display on a swap trial could contain a yellow horizontal bar in the upper quadrant and a red vertical bar in the lower quadrant. If pigeons had bound the features (color, orientation, and location) of the first display, then they should notice that the compound relations among these features in the second display had changed, and thus, should select the key associated with a display change. Pigeons did not respond to these swap trials as they did to change trials, and instead responded to them as if they were identical displays. Thus, pigeons failed to show evidence of feature binding. Human participants learning the same procedure did show evidence of feature binding at test.

Perhaps the task was ill suited to promoting feature binding in pigeons because binding of feature information was not necessary for accurate performance in the task. While all three features were changed on display-change trials, pigeons could have solved the task by attending to only one of the three features. Thus, the ability to use a single feature to solve the discrimination may have prevented feature binding.

The other prior study to look at binding in pigeons used a procedure designed to measure binding errors (Katz et al., 2010). Pigeons learned to detect a target compound when presented with a non-target compound within the same trial. The compounds were presented simultaneously on some trials and sequentially on others. Trials on which neither compound was a target were included to test for binding errors. For example, if the target compounds were a red ' $U$ ' shape and a yellow ' $T$ ' shape, then a test trial might consist of the sequential presentation of a yellow ' $U$ ' shape followed by a red ' $T$ ' shape. If pigeons responded to these non-target compounds similarly to how they respond to the targets, this would be scored as a binding error. Such an error indicates that the pigeon had formed an illusory conjunction of target color with target shape (Treisman, 1996). Some support for binding errors was found in some of the subjects during the initial stage of training, but disappeared by the end of training. Binding errors were mostly found in the sequential presentation of non-target compounds, and very little on trials on which the non-target compounds were presented simultaneously. The complexity of the task, which involved mixed training on single-item and multiple-item discriminations, and the nuanced phenomena that emerged as a result, such as recency effects and the transient nature of binding effects mitigate the strength of evidence this study can provide for binding in pigeons. 
The prior procedures with pigeons utilized tasks of perceptual feature binding and met with limited success. Perhaps a different approach, using a task of memory feature binding would prove more successful. We developed a procedure using an SRT procedure to encourage feature binding in pigeons. SRT procedures have a long history of use in the study of implicit and procedural learning in humans (Nissen \& Bullemer, 1987) and animals (Christie \& Dalrymple-Alford, 2004; Froehlich, Herbranson, Loper, Wood, \& Shimp, 2004). Sequence learning in SRT tasks is thought to involve multiple brain regions, from sensory to memory to motor (Keele et al, 2003; Robertson, 2007). In humans, SRT tasks have also been used to study whether object identity, location, or both can be learned. In studies of contextual cueing by which object identity or location can be repeated in sequence, Endo and Takeda (2004; see also Higuchi et al., 2016; Jiang \& Song, 2005) found evidence that each cue can be learned on its own, but when combined, only the more salient cue-typically the cue that is more task relevant-is learned. Similar studies using cues from different modalities to study multisensoryintegration have found synergistic effects of having both visual and tactile or visual and auditory cues. Moreover, multisensory congruency supports explicit awareness of sequence information (Silva et al., 2017). Thus, while there is conflicting information as to whether having multiple cues would aid in sequence learning, the SRT procedure provides a plausible alternative to the perceptual binding tasks described above for the study of memory binding in the pigeon.

The task was simple and was designed to directly manipulate attention to feature conjunctions. In each session, a repeating sequence of four objects ( $A, B, C, \& D)$ were consecutively displayed in one of four locations $(1,2,3, \& 4)$ on a touchscreen. The first peck to the object removed it from the screen and the next object was displayed. Reinforcement was periodically delivered following an object peck. Thus, all the pigeon needed to do to earn reinforcement was to peck at an object as soon as it appeared. There were twelve birds placed on this task, and each bird went through two rounds of training. In each round of training, a different set of four objects was used (Figure 1) and each pigeon was assigned to one of four treatment groups (see Figure 2 for the four 4-object sequence conditions used in each round). In Group Location-Only Training, the location at which an item appeared repeated in each sequence, but the objects appeared in random order across sequences. In Group Object-Only Training, the objects were presented in a consistent sequence, but the locations at which they appeared were randomized across sequences. In Group Both Training, both object and location appeared in a repeating consistent sequence (e.g., arrow in upper left, shield in upper right, 
circle in lower left, arrow in lower right). Finally, in Group Random Training, both the objects and locations appeared in randomized sequences.

What did we hope to accomplish with this sequence learning procedure? It is well known that, like humans, pigeons are sensitive to repeated sequences which they can readily learn (Froehlich et al., 2004; Herbranson \& Stanton, 2011) and can readily learn regularities in sequence structure such as those that underlie artificial grammars (Herbranson \& Shimp, 2008). We hypothesized that by repeating sequences, the pigeons in our study should attend to the serial order of information in the repeated sequences. When serial order consisted of repeated locations, pigeons should attend to location. When it consisted of objects, they should attend to object identity. When the order of both object and location were repeated consistently, then pigeons should attend to both. We additionally hypothesize that consistently repeating object and location information together during training should increase attention to both features, and thereby promote memory feature binding. That is, the pigeons should form object-location associations. Alternatively, if one of the features, such as location, is more salient than the other, such as object, as has been found in human studies (Endo \& Takeda, 2004; Higuchi et al., 2016; Jiang \& Song, 2005), then we might hypothesize that the same overshadowing of object information by location information will be observed in pigeons, in which case memory binding based on object-location associations should be prevented.

To test for sequence learning in Groups Location-Only Training and Object-Only Training, and object-location binding in memory in Group Both Training, nonreinforced probe sequences were delivered. There were four types of probe sequence (Figure 3). Location probes switched the order of locations between two of the four displays within the sequence. Object probes swapped the order of objects between two of the four displays. The remaining two types of probe sequence involved swapping both object and location between two of the displays within the sequence. For Unbound probes, this involved presenting one of the objects at a different location than typical in a consistent sequence condition (see lower right panel of Figure 3 for an example). This swap resulted in the breaking of any prior object-location association for Group Both Training. Finally, on Bound probes the order of two of the displays were swapped within-sequence (see upper left panel of Figure 3 for an example). By swapping the order of displays, the object-location association was preserved, though now presented out of sequence. 
The probe sequences systematically broke sequence consistency of object, location, or both for subjects that had received those features in a consistent sequence during training. Thus, if pigeons in Group Both Training had bound object-location information during training, as hypothesized above, then any change that disrupts object-location binding should result in slower response times (RTs) to these displays compared to training displays in which objectlocation information is preserved. That is, there should be an RT cost on probe sequences if binding is violated, but only for pigeons that had bound object and location information. The object-location relationship is broken on Object, Location, and Unbound probes. Only the Bound probes preserves the object-location association, and thus we predicted much less RT cost on Bound probes by birds in Group Both Training. RT cost was measured as a percent increase in mean RT on probe sequences relative to training (baseline) sequences.

Additional predictions can be made about pigeons in the other three treatment groups. The order of locations was consistent during training for Group Location-Only Training, therefore switching the order on Location and Bound probes should induce an RT cost for those birds, while switching the order of objects should not. Likewise, switching object order on Object and Bound probes should induce an RT cost for pigeons trained on object consistent displays (Group Object-Only Training), while no RT cost should be observed on Location switch probes. Finally, pigeons in Group Random Training experienced no consistency in sequence information during training, and thus were not expected to show any RT costs on any probe sequence.

\section{Experiment 1}

In Experiment 1, pigeons were consecutively trained on two 4-item sequences that repeated throughout each session: Location consistent, Object consistent, Both consistent, or Random. Each of the two sequences were trained in separate rounds of training and testing (described below). Once responding had stabilized at asymptotic rates, probe sequences were presented. Probe sequences could disrupt various aspects of sequence information, such as location, object, or both.

\section{Methods}

Subjects. Twelve pigeons (Columba livia) served as subjects. They had previously participated in a variety of open-field and touchscreen experiments, but were naive with respect to the stimuli and sequence-learning task used in this experiment. Pigeons were maintained at $80-85 \%$ of their free-feeding weights, and were individually housed in a colony with a 12-h 
light-dark cycle. They had free access to water and grit in their home cage. Experimental procedures occurred during the light portion of the cycle.

Apparatus. Training and testing was conducted in a flat-black Plexiglas chamber $(38 \mathrm{~cm}$ wide $\times 36 \mathrm{~cm}$ deep $\times 38 \mathrm{~cm}$ high). All stimuli were presented by computer on a color LCD monitor (NEC MultiSync LCD1550M) visible through a $23.2 \mathrm{~cm} \times 30.5 \mathrm{~cm}$ viewing window in the middle of the front panel of the chamber. The bottom edge of the viewing window was $13 \mathrm{~cm}$ above the chamber floor. Pecks to the monitor were detected by an infrared touchscreen (Carroll Touch, Elotouch Systems, Fremont, CA) mounted on the front panel. A 28-V houselight located in the ceiling of the box was illuminated at all times, except when an incorrect choice was made. A servo-driven food hopper (produced in house) was located behind the front panel with its access hole flush with the floor centered below the touchscreen. All experimental events were controlled and recorded with a Pentium III-class computer (Dell, Austin, TX). A video card controlled the monitor in the SVGA graphics mode (800 pixels $\times 600$ pixels). The stimuli used in this procedure are similar to those used by (Blaisdell $\&$ Cook, 2005). The stimuli and $3 \times 3 \mathrm{~cm}$ screen locations where they could be presented are shown in Figure 1 separately for each round of testing. For round 1, these locations were arranged $8 \mathrm{~cm}$ from the top, $7 \mathrm{~cm}$ from the sides, $4 \mathrm{~cm}$ from the bottom of the screen. For round 2, these locations were arranged by rotating the $2 \times 2$ grid of response boxes by 45 degrees such that one location was centered horizontally along the upper part of the screen, another centered horizontally along the lower part, and the remaining two centered vertically along the left and right sides of the screen. Each response box was surrounded by a white border to demark the location where the stimuli could appear.

Procedure. Pigeons had previously been trained to eat grain from the food hopper (magazine training), so training began immediately with a mixed autoshaping/instrumental shaping procedure using the sequence-learning task. Each trial consisted of the presentation of one of four objects $(A, B, C, \& D)$ at one of four locations $(1,2,3, \& 4)$ on the screen. Each bird was randomly assigned to one of four conditions in which presentation order could occur in a repeating sequence. For birds in Group Location-Only Training, the locations were ordered in a repeating sequence $(1 \rightarrow 2 \rightarrow 3 \rightarrow 4)$ but object order was randomized in blocks of 4 trials (Figure 2, top right panel). For birds in Group Object-Only Training, the objects were presented in a recurring sequence $(A \rightarrow B \rightarrow C \rightarrow D)$, but location order was randomized in blocks of 4 trials (Figure 2, bottom left panel). Items were presented on the screen in one of the array locations, one at a time, and remained on screen for either $30 \mathrm{~s}$ or until pecked. Once the $30 \mathrm{~s}$ had 
elapsed or the item had been pecked, whichever came first, the item was removed and the hopper was raised for $3 \mathrm{~s}$. As the hopper was lowered, the next item was presented. The object and location of each item depended on treatment group. For subjects in Group Location-Only Training, the locations where objects could be presented were presented in a consistent, repeating order, but the order of objects was randomized across sequences (e.g., A1, B2, C3, D4, C1, A2, D3, C4, etc.). For subjects in Group Object-Only Training, the objects were presented in a consistent, repeating order, but location order was randomized across sequences (e.g., A1, B2, C3, D4, A3, B4, C1, D2, etc.). For subjects in Group Both Training, both object and location order were consistent and repeated across sequences (e.g., A1, B2, C3, D4, A1, B2, C3, D4, etc.). For subjects in Group Random Training, the location and object order was randomized (e.g., A1, B2, C3, D4, B3, A4, D2, C1, etc.). Randomization was done by scrambling the four objects and/or locations for each 4-item sequence.

Once subjects were responding on a majority of trials (>80\%), the 30 -s trial time limit was discontinued, and the item remained on screen until pecked. During this phase the interstimulus interval (ISI) was randomly selected from a range between 5 and $15 \mathrm{~s}$. As peck rate increased, the ratio of rewarded to unrewarded trials was increased until reinforcement was delivered on average every $20^{\text {th }}$ trial. Any trial that followed a rewarded trial was excluded from analysis to avoid artefacts introduced by the consummatory response. Test sessions began once peck rates had stabilized.

Test sessions were identical to training sessions except that 20 of the 90 training sequences were replaced with non-reinforced probe sequences. Four types of probe sequence were used for all subjects regardless of their training condition (see an example of each in Figure 3). Each probe sequence consisted of a change in the 4-item sequence. On Object probe sequences, the order of two of the objects in the sequence were swapped with each other without altering the sequence of locations. On Location probe sequences, the order of two locations in the sequence were swapped without altering the sequence of objects. On Bound probe sequences, the order of two objects and locations were swapped such that the objectlocation association that occurred during training was maintained (e.g., instead of A1, B2, C3, D4, the subject might receive A1, C3, B2, D4, with the boldface indicating the swapped items). On Unbound sequences, one of the items in the sequence consisted of a repeat of an object and location used elsewhere in the sequence such that the object-location association differed from that used in the training sequence (e.g., instead of $A 1, B 2, C 3, D 4$, the subject might receive $A 1, D 3, C 3, D 4$, with the boldface indicating the probe item). At least three training 
sequences occurred before a probe sequence was scheduled for delivery, and the first probe sequence was not presented until after the first ten training sequences in the test session had occurred.

After collecting probe data in round 1, pigeons were reassigned to new treatment groups in counterbalanced fashion, such that one bird from each treatment condition was assigned to one of the other treatment conditions (e.g., for Group Both, one bird was reassigned to Group Location, another to Group Object, and the third to Group Random Training). After reassignment, birds received a new round of training and testing using the same procedures as for round 1, with the exception that new stimuli and screen locations were used (Figure 1, right panel).

RT from stimulus onset to subject peck in the response box was recorded on each trial and served as our dependent measure.

Data Analysis. Baseline and Probe sequence data were collapsed across all sessions for the last 100 probe sequences, separately for each subject and for each replication, resulting in a final $n=6$ per treatment group. RT cost on probe sequences was normalized for each subject by subtracting mean Baseline RT of the preceding baseline sequence from mean Probe sequence RT on the subsequent probe sequence, and this difference was then divided by mean Baseline RT and multiplied by 100 to yield a percentage change in RT. For example, if the mean Baseline RT was $1 \mathrm{~s}$ and the mean Probe RT was $1.5 \mathrm{~s}$, RT cost would be calculated as $(1.5-1) / 1=.5 \times 100=50 \%$.

While mean Probe sequence RTs were compared to mean RTs of the preceding baseline sequences, there were a few restrictions placed on baseline RT selection for this calculation. Mean baseline RTs were only taken from baseline sequences in which reinforcement had not been delivered. Data from any baseline and probe sequence on which the first response was outside the response box in which the stimulus was present were excluded from analysis.

\section{Results \& Discussion}

A mixed ANOVA conducted on the percentage difference RT with Group as a betweengroup factor and Probe type as a repeated measure revealed main effects of Group, $F(3,20)=$ $7.80, p<0.01$, and Probe type, $F(3,60)=3.17, p<0.05$, as well as a Group $x$ Probe Type interaction, $F(9,60)=5.24, p<0.01$ (Figure 4). Planned comparisons were performed to test our 
specific predictions. In Group Location-Only Training, RT cost on object probe sequences was lower than on the other three probe sequences where location was changed, smallest $F(9,20)=$ 9.02, $p<0.01$. In Group Both Training, RT cost was higher on Unbound probe sequences than on all other probe sequences, smallest $F(1,20)=15.90, p<0.01$. Furthermore, RT cost was lower on Bound probe sequences than all other probe sequences, smallest $F(1,20)=11.16, p<$ 0.01 . These results support the hypothesis that pigeons in Group Both Training, that had received training with both location and object in a consistent sequence, had perceptually bound object and location information of the items. No significant differences were found between probe types in Group Object-Only Training or Group Random Training, $F_{S}(1,20)<1.0$.

Group Random Training failed to show any RT costs on probe trials. Thus, this group serves as a useful control in that no RT costs were found under training and testing conditions in which they were not expected. No effects were predicted because sequence order during training was random for both object and location information. Switching the order on probe trials was undetectable because order was already random during training.

For Group Object-Only Training, only the order of objects was consistent during training, so we predicted that switching object order on Object probes should produce an RT cost. No RT cost was observed, however, which suggests that birds in this group did NOT encode the order of object during training.

For Group Location-Only Training, location order was encoded as evidence by the higher RT costs on probe types on which location order was changed. Thus, the failure for pigeons in Group Object-Only Training to encode object order was not due to fundamental limitations with the procedure itself. Instead, it likely reflects object information being less salient than location information. In retrospect this makes sense because responding is motivated by food. Thus, any predictive information that could facilitate rapid responding should receive greater attention. Location information does provide predictive information as to where the next item in the list will appear, whereas object information cannot be used to reduce response times because all the subject need do is peck at an item as soon as it appears. The identity of the object is irrelevant.

Nevertheless, in probes in which only the order of objects was changed (Object probe sequences), RT increased only for pigeons in Group Both Training. Group Both Training is the only group for which object-location information was correlated during training. The increased RT on object probe sequences in Group Both Training suggests that the object and location had 
become perceptually bound during training, such that any probe sequence that broke this association resulted in an RT cost. Correlating object and location consistency had the benefit of increasing the salience of object order information, probably due its being bound to location information which pigeons did encode even in the absence of a consistent object order (i.e., in Group Location-Only Training). The sequence learning procedure therefore appears to have enabled recruitment of attentional processing of location information. Furthermore, when object information was correlated with location information, the increased attention to object order enabled object-location feature binding similar to what has been reported in primates.

\section{Experiment 2}

The primary results of Experiment 1 were a) the finding that birds encoded location order when it was consistent within sequence, b) but failed to encode object order when it was consistent within sequence; c) when both object and location order were consistent within sequence, then pigeons encoded both object and location order; and d) consistently repeating object and location order together within sequence resulted in object-location binding.

By ordering both location and object in a consistent sequence, the same object always appeared at the same location. This could enable the establishment of object-location associations. While the association between a location and an object may be learned through such repeated pairings, it was not clear if such pairings were sufficient for learning of objectlocation associations. The sequential aspect to this task offered many additional cues to increase the salience of the object-location pairings, such as local statistical information between adjacent items (Froehlich et al., 2004). In the study by Froehlich et al using a serial reaction time task, response times were faster to repeated lists compared to randomly ordered item lists. This shows that pigeons benefit from item repetition within lists to facilitate attention to the next anticipated item. In Experiment 2, we address the question, was having consistent object-location correlation sufficient for binding, or was sequence consistency also necessary for object-location binding? To answer this question, we trained six new pigeons on the sequence learning procedure. Three of the pigeons received the same training as Group Both Training received in the first round of Experiment 1. The other three pigeons also received training on stimuli for which the same object always appeared at the same location, but presentation order was randomized across sequences (Figure 5, left panel), thus, we call this treatment group the Association condition because it could allow for the establishment of an object-place association despite randomization of within-sequence order. After peck rates had 
stabilized, pigeons received the same series of probe tests as described in Experiment 1. If consistency between object and location was sufficient to support feature binding, then Groups Both Training and Association should both show the same pattern of RT cost effects on probe trials as was shown for Group Both Training in Experiment 1. Alternatively, if maintaining a consistent sequence for both object and location is necessary for binding, then we only expect to replicate the pattern of RT cost effects in Group Both Training, but not in Group Association.

\section{Methods}

Subjects. The subjects were six new pigeons (Columba livia) from the same colony as those used in Experiment 1, but naive with respect to the current experimental stimuli and procedure.

Apparatus. The apparatus was the same as in Experiment 1.

Procedure. Pigeons were randomly assigned to one of two groups, Both Training and Association. Training proceeded as in Experiment 1 using the stimuli and locations used in round 1 (Figure 1, left panels). Group Both Training received the same treatment as described for Group Both Training in round 1 of Experiment 1. Group Association received training on a similar procedure as Group Both Training in which each object could only appear at a specific location with the exception that item order was randomized within-sequence (e.g., A1, B2, C3, D4, B2, D4, A1, C3, etc., Figure 5, left panel).

\section{Results \& Discussion}

Inspection of individual bird data revealed that two of the pigeons in Group Both Training replicated the pattern of results from Experiment 1, while a third pigeon did not replicate this pattern of results and showed no evidence of binding, despite training for Group Both Training. Thus, data from this non-replicating subject were excluded from analysis.

A mixed ANOVA conducted on the percentage difference RT with Group as a betweengroup factor and Probe type as a repeated measure revealed an effect of Group, $F(1,3)=$ 128.15, $p<0.01$, but no effect of Probe type, $F(3,9)=2.74, p>0.10$, nor a Group $x$ Probe type interaction, $F(3,9)=2.08, p>0.10$ (Figure 5, right panel). Planned comparisons were performed to test our specific predictions. For Group Both Training, compared to Unbound probes, RT cost was lower to Bound probes, $F(1,3)=13.30, p<0.05$, marginally lower to Object probes, $F(1,3)$ $=10.04, p=0.051$, but not lower to Location probes, $F(1,3)=4.20, p>1.0$. RT costs did not 
differ among Bound, Object, and Location probes, largest $F(1,3)=1.29, p>0.30$. For Group Association, RT cost did not differ among any probe type, largest $F(1,3)=1.53, p>0.30$.

We replicated the difference found in Group Both Training in Experiment 1 between Both Unbound and Bound probes. We also came close to replicating the difference between Unbound probes and Object probes observed in Experiment 1, while we failed to replicate the difference between Unbound and Location probes. Some of the replication failures likely result from the small $\mathrm{n}$ in Group Both Training in Experiment 2. For the new condition, Group Association, none of the probe trials differed from each other. In particular, the lack of a difference in RT cost on probe trials that preserved the object-location association (Bound probes) versus those on which the object-location association was broken (Unbound probes), and that there was no RT cost on either of these probe sequences suggests that the pigeons in this treatment group did not bind object and location information during training. Thus, it appears that merely correlating object and location information was not sufficient for memory feature binding, and that having a consistent sequence during training for both object and location information was necessary for object-location binding to occur. These results point to the importance of local statistical information in driving attentional processes necessary for feature binding (Froehlich et al., 2004).

Sequence consistency in Group Both Training may have facilitated object-location binding in several ways. Since location order was consistent, pigeons could predict where each next object would appear based on the location of the previous object. This could allow the pigeon to begin attending to the anticipated location prior to the onset of the object. As a result, when the object finally appears, the pigeon is better prepared to process that object information. Increased processing of the object prior to its being pecked (and thus disappearing from the screen) could facilitate object-location binding. Since the location of each object could not be anticipated by pigeons in Group Association, objects did not benefit from this extra processing, and thus failed to be bound with location information, despite the fact that each object could only appear (and thus be associated with) a particular location. Processing of visual information has been shown to similarly benefit from other techniques that increase anticipation of information, such as priming in visual search (D. S. Blough, 2000; P. M. Blough, 1989; (Bond \& Kamil, 1999; Cook et al., 1997).

\section{General Discussion}


Pigeons were presented with a repeated sequence of four objects at four locations. For different groups of pigeons in Experiment 1, the objects, locations, or both could be repeated in a consistent or random order. To test for encoding of ordered sequences, probe tests were conducted in which object order, location order, or both could be disrupted. These tests revealed that pigeons had encoded the location order in both Group Location-Only Training and Group Both Training. There was no evidence that object order was encoded in Group ObjectOnly Training, but object order was encoded in Group Both Training. It is possible that object order was encoded by birds in Group Object-Only Training, but they might not have been able to use this knowledge to respond more quickly. Knowledge of upcoming objects is less likely to produce robust RT facilitation than knowledge of upcoming locations, the latter allowing for preparatory responding. Moreover, for pigeons in Group Both Training, changing the object and location order such that the object-order relationship used in training was preserved had much less impact, evidenced by low RT costs, compared to changing the object and location order such that the object-location relationship used in training was broken. This difference reflects object-location binding by pigeons in Group Both Training. In Experiment 2, when objectlocation association was maintained, but sequence order randomized (Group Association), no binding was found. Thus, feature binding depended on maintaining a consistent item sequence order. This is consistent with other reports using similar procedures that show that local statistical information facilitates anticipatory responses in pigeons (Froehlich, 2004). By facilitating anticipatory responding, attention to feature conjunctions, such as between object and location, is enhanced, thereby facilitating feature binding.

This is the first evidence for strong and lasting memory feature binding in pigeons. Notably, Treisman suggested location binding in which objects are bound to their location may be the most basic binding problem brains are designed to solve (Treisman, 1996). Presenting objects and locations in a consistent order within sequence was necessary for their binding. Prior studies have shown that pigeons will naturally (i.e., without explicit reinforcement) encode sequences of locations (Froehlich et al., 2004; Herbranson \& Stanton, 2011; Herbranson, Xi, \& Trinh, 2014), though they can also learn sequences through reinforcement (Garlick, Fountain, \& Blaisdell, 2016). Perhaps prior studies that failed to show lasting feature binding resulted from procedures that failed to sufficiently increase the salience of, and thereby attention to feature conjunctions.

This is the first report of strong and lasting memory binding in pigeons. Past attempts at identifying object-location binding in pigeons have used very different procedures. Lazareva and 
Wasserman (2016) found no evidence of object-location binding in pigeons using a change detection task. Their procedure used presentations of displays made from multiple colored lines at varying orientations. Though birds learned to classify displays as different if they contained novel orientations or colors, trials in which only one or two of the three properties were altered between slides were treated as being the same. This was taken as evidence that pigeons did not bind feature information about the objects. Nevertheless, as discussed in the Introduction, as they were trained, pigeons could easily solve their task by attending to only one feature at a time. There was no need to attend to multiple dimensions simultaneously. Binding appears to require attention to multiple features so that they can be integrated into a configural object representation. Likewise, the positive evidence for feature binding in pigeons reported by Katz et al. (2010) was fleeting and not found in all pigeons tested. Thus, their procedure may have initially caused the pigeons to attend to feature conjunctions, behavioral control by these conjunctions waned with further training.

Even in our procedure, reinforcement did not depend on object-location encoding, and thus the same criticism could be directed here. Nevertheless, pigeons trained in Group Both Training, where items with consistent object-location associations were presented in a consistent sequence, did show evidence of object-location memory binding, thus obviating this criticism. Apparently, something about our procedure worked, where others have failed or met with limited success. Perhaps it had to do with the fact that performance was facilitated by attending to location information. As a result, conjunctions between object and location features (Experiments 1 and 2, Group Both Training) may have increased in salience compared to conditions in which object-location conjunctions were present, but location order was random (Experiment 2, Group Association).

These experiments add to the growing literature on the pigeon visual system. The architecture of the higher-level pallial structures of the avian and mammalian brain differ markedly. Like other classes of vertebrates, the avian pallium is organized as a network of nucleated circuits. The mammalian pallium, however, consists of a laminar isocortex. Nevertheless, despite these architectural differences, the functional connectivity of bird and mammal pallium are quite similar Reiner (2004). Thus, the avian brain is a useful animal model for studying human cognitive neuroscience (Clayton \& Emery, 2015). Furthermore, like the mammalian visual system, the avian visual system is adapted to the rapid processing of visual input (Cook, 2000). This involves the operation of similar mechanisms of early vision and search, such as the segregation of visual input through separate channels, and their 
recombination at higher-order brain areas (Wylie et al., 2009). For example, features such as color, shape, and size have been shown to be processed independently (Cook, 1992b; Cook, Cavoto, \& Cavoto, 1996), and reintegrated to meet task demands such as visual search for targets defined by specific feature conjunctions (Cook et al., 1996; George \& Pearce, 2003). The ability to discriminate target items based on feature conjunctions is a necessary precursor to feature binding. Feature binding goes beyond feature conjunctions in that binding is automatic and plays a fundamental role in object learning and recognition. By using an implicit learning task involving ordered sequences, our experiments provide strong evidence for the automatic binding of features in pigeons. 


\section{References}

Bitterman, M. E. (1975). The comparative analysis of learning. Science, 188(4189), 699-709. https://doi.org/10.1126/science.188.4189.699

Blaisdell, A. P., \& Cook, R. G. (2005). Two-item same-different concept learning in pigeons. Learning \& Behavior, 33(1), 67-77.

Blough, D. S. (2000). Effects of priming, discriminability, and reinforcement on reaction-time components of pigeon visual search. Journal of Experimental Psychology: Animal Behavior Processes, 26(1), 50-63. https://doi.org/10.1037/0097-7403.26.1.50

Blough, D. S., \& Blough, P. M. (1997). Form perception and attention in pigeons. Animal Learning \& Behavior, 25(1), 1-20. https://doi.org/10.3758/BF03199020

Blough, P. M. (1989). Attentional priming and search images in pigeons. Journal of Experimental Psychology: Animal Behaviour Processes, 15(4), 211-223.

Blough, P. M. (1991). Selective Attention and Search Images in Pigeons. Journal of Experimental Psychology: Animal Behavior Processes, 17(3), 292-298. https://doi.org/10.1037/0097-7403.17.3.292

Bond, A. B., \& Kamil, A. C. (1999). Searching image in blue jays: Facilitation and interference in sequential priming. Animal Learning and Behavior, 27(4), 461-471. https://doi.org/10.3758/BF03209981

Christie, M. A., \& Dalrymple-Alford, J. C. (2004). A new rat model of the human serial reaction time task: Contrasting effects of caudate and hippocampal lesions. Journal of Neuroscience, 24, 1034-1039.

Clayton, N. S., \& Emery, N. J. (2015). Avian models for human cognitive neuroscience: A proposal. Neuron, 86(6), 1330-1342. https://doi.org/10.1016/j.neuron.2015.04.024

Cook, R. G. (1992a). Acquisition and transfer of visual texture discriminations by pigeons. Journal of Experimental Psychology: Animal Behavior Processes, 18(4), 341-353. https://doi.org/10.1037/0097-7403.18.4.341

Cook, R. G. (1992b). Dimensional organization and texture discrimination in pigeons. Journal of 
Experimental Psychology: Animal Behavior Processes, 18(4), 354-363.

https://doi.org/10.1037/0097-7403.18.4.354

Cook, R. G. (2000). The comparative psychology of avian visual cognition. Current Directions in Psychological Science, 9(3), 83-89. https://doi.org/10.1111/1467-8721.00066

Cook, R. G. (2001). Avian Visual Cognition. [On-line]. Available: pigeon.psy.tufts.edu/avc/

Cook, R. G., Cavoto, B. R., Katz, J. S., \& Cavoto, K. K. (1997). Pigeon perception and discrimination of rapidly changing texture stimuli. Journal of Experimental Psychology: Animal Behavior Processes, 23(4), 390-400. https://doi.org/10.1037/0097-7403.23.4.390

Cook, R. G., Cavoto, K. K., \& Cavoto, B. R. (1996). Mechanisms of multidimensional grouping, fusion, and search in avian texture discrimination. Animal Learning \& Behavior, 24(2), 150 167.

Cook, R. G., Katz, J. S., \& Blaisdell, A. P. (2012). Temporal properties of visual search in pigeon target localization. Journal of Experimental Psychology: Animal Behavior Processes, 38(2), 209-216. https://doi.org/10.1037/a0026496

Endo, N., \& Takeda, Y. (2004). Selective learning of spatial configuration and object identity in visual search. Perception \& psychophysics, 66(2), 293-302.

Froehlich, A. L., Herbranson, W. T., Loper, J. D., Wood, D. M., \& Shimp, C. P. (2004). Anticipating by pigeons depends on local statistical information in a serial response time task. Journal of Experimental Psychology: General, 33(1), 31-45. https://doi.org/10.1037/0096-3445.133.1.31

Garlick, D., Fountain, S. B., \& Blaisdell, A. P. (2016). Serial pattern learning in pigeons: RuleBased or associative? Journal of Experimental Psychology: Animal Learning and Cognition, 43(1), 30-47. https://doi.org/10.1037/xan0000109

George, D. N., \& Pearce, J. M. (2003). Discrimination of structure: II. Feature binding. Journal of Experimental Psychology: Animal Behavior Processes, 29(2), 107-117. https://doi.org/10.1037/0097-7403.29.2.107

Herbranson, W. T., \& Shimp, C. P. (2008). Artificial grammar learning in pigeons. Learning \& Behavior, 36(2), 116-137. https://doi.org/10.3758/LB.36.2.116 
Herbranson, W. T., \& Stanton, G. L. (2011). Flexible serial response learning by pigeons (Columba livia) and humans (Homo sapiens). Journal of Comparative Psychology, 125(3), 328-340. https://doi.org/10.1037/a0023299

Herbranson, W., Xi, P. M., \& Trinh, Y. T. (2014). Spatial variability in serial response learning and performance by pigeons (Columba livia). International Journal of Comparative Psychology, 27(2), 280-294.

Higuchi, Y., Ueda, Y., Ogawa, H., \& Saiki, J. (2016). Task-relevant information is prioritized in spatiotemporal contextual cueing. Attention, Perception, \& Psychophysics, 78(8), 23972410.

Husband, S. \& Shimizu, T (2001). Evolution of the avian visual system. In R. G. Cook (Ed.), Avian Visual Cognition [On-line]. Available: pigeon.psy.tufts.edu/avc/husband/

Jiang, Y., \& Song, J. H. (2005). Hyperspecificity in visual implicit learning: learning of spatial layout is contingent on item identity. Journal of Experimental Psychology: Human Perception and Performance, 31(6), 1439-1448.

Kastner, S., De Weerd, P., Desimone, R., \& Ungerleider, L. G. (1998). Mechanisms of directed attention in the human extrastriate cortex as revealed by functional MRI. Science, 282(5386), 108-111. https://doi.org/10.1126/science.282.5386.108

Katz, J. S., Cook, R. G., \& Magnotti, J. F. (2010). Toward a framework for the evaluation of feature binding in pigeons. Behavioural Processes, 85(3), 215-225. https://doi.org/10.1016/j.beproc.2010.07.017

Keele, S. W., Ivry, R., Mayr, U., Hazeltine, E., \& Heuer, H. (2003). The cognitive and neural architecture of sequence representation. Psychological Review, 110(2), 316-339.

Lazareva, O. F., \& Wasserman, E. A. (2016). No evidence for feature binding by pigeons in a change detection task. Behavioural Processes, 123, 90-106. https://doi.org/10.1016/j.beproc.2015.09.007

Luck, S. J., Chelazzi, L., Hillyard, S. A., \& Desimone, R. (1997). Neural mechanisms of spatial selective attention in Areas V1, V2, and V4 of macaque visual cortex. Journal of Neurophysiology, 77(1), 24-42. https://doi.org/10.1152/jn.1997.77.1.24

Nissen, M. J., \& Bullemer, P. (1987). Attentional requirements of learning: Evidence from 
performance measures. Cognitive Psychology, 19(1), 1-32.

Reiner, A., Perkel, D. J., Bruce, L. L., Butler, A. N. N. B., Csillag, S., Kuenzel, W., ... Ball, G. F. (2004). Revised nomenclature for avian telencephalon and some related brainstem nuclei. Journal of Comparative Neurology, 473(3), 377-414. https://doi.org/10.1002/cne.20118

Robertson, E. M. (2007). The serial reaction time task: implicit motor skill learning?. Journal of Neuroscience, 27(38), 10073-10075.

Silberberg, A., \& Kearns, D. (2009). Memory for the order of briefly presented numerals in humans as a function of practice. Animal Cognition, 12(2), 405-407.

\section{https://doi.org/10.1007/s10071-008-0206-8}

Silva, A. E., Barakat, B. K., Jimenez, L. O., \& Shams, L. (2017). Multisensory congruency enhances explicit awareness in a sequence learning task. Multisensory Research, 30(7-8), 681-689.

Treisman, A. (1996). The binding problem. Current Opinion in Neurobiology, 6, 171-178. https://doi.org/10.1016/S0959-4388(96)80070-5

Treisman, A. M., \& Gelade, G. (1980). A feature-integration theory of attention. Cognitive Psychology, 12, 97-136. https://doi.org/10.1016/0010-0285(80)90005-5

Wylie, D. R. W., Gutierrez-Ibanez, C., Pakan, J. M. P., \& Iwaniuk, A. N. (2009). The optic tectum of birds: Mapping our way to understanding visual processing. Canadian Journal of Experimental Psychology, 63(4), 328-338. https://doi.org/10.1037/a0016826 
Stimuli used in round 1

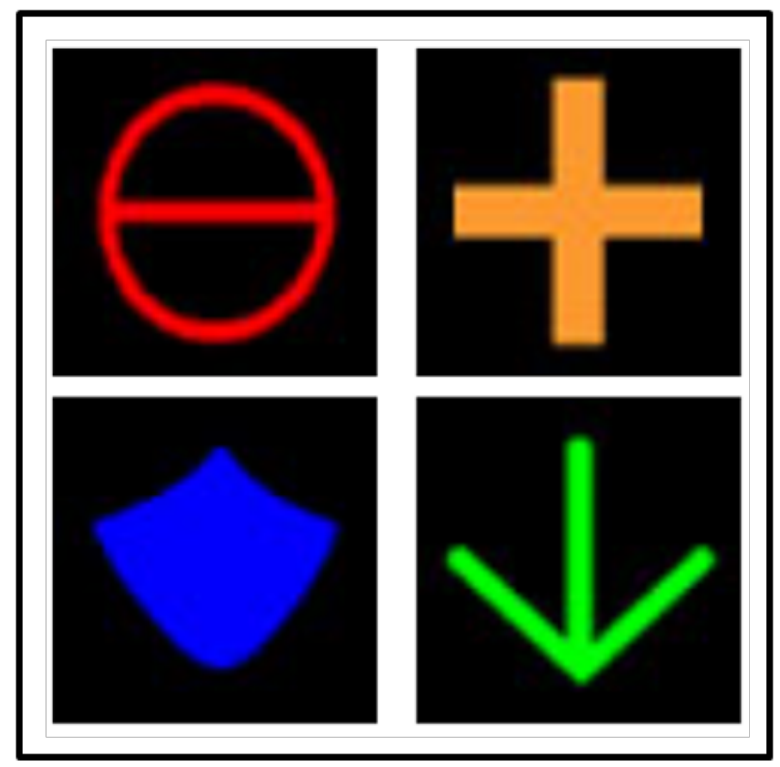

Screen locations used in round 1

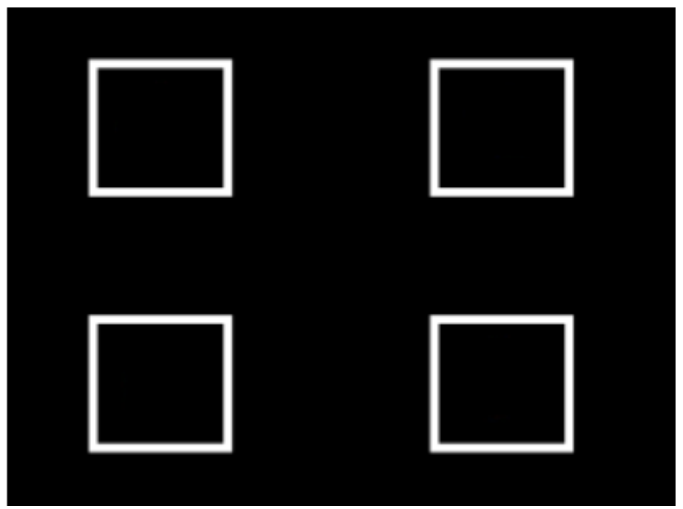

\section{Stimuli used in round 2}

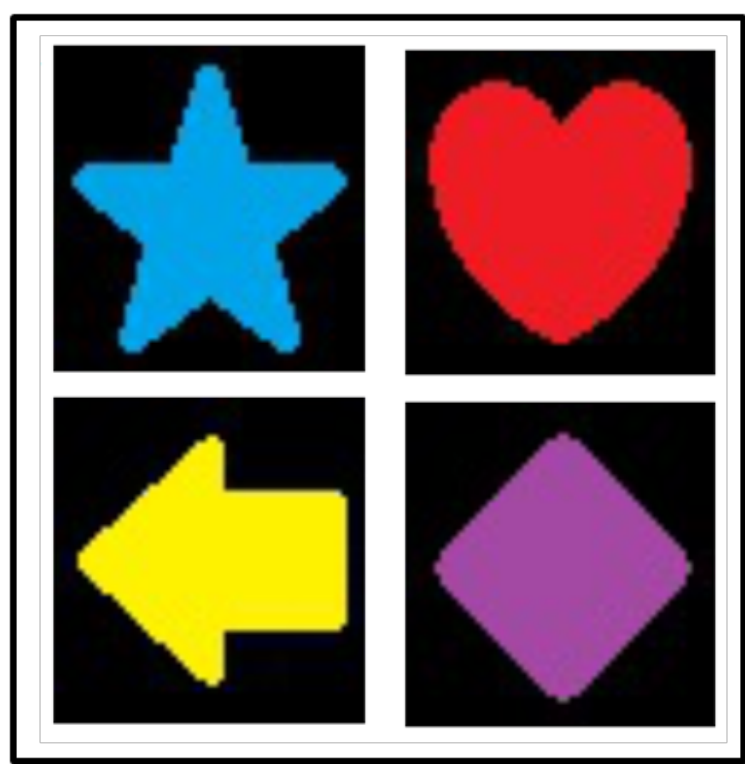

Screen locations used in round 2

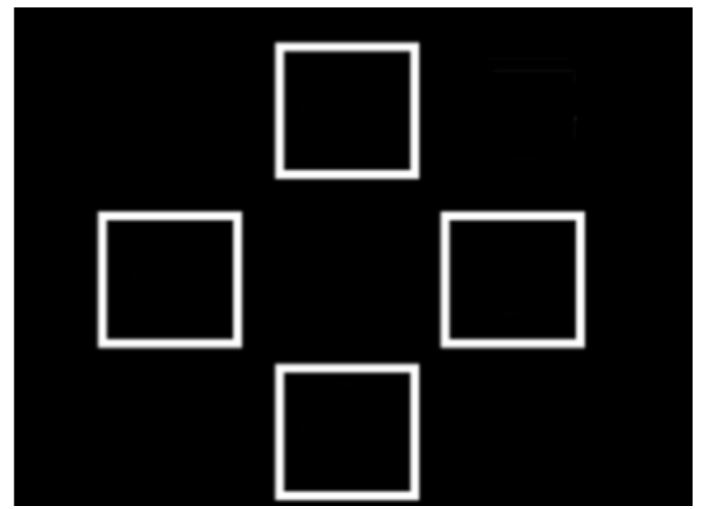

Figure 1. Stimulus sets and screen response locations used in Experiments 1 and 2. Left panels show stimuli and screen locations for round 1 of Experiment, and Experiment 2. Right panels show stimuli and screen locations for round 2 of Experiment 1. 


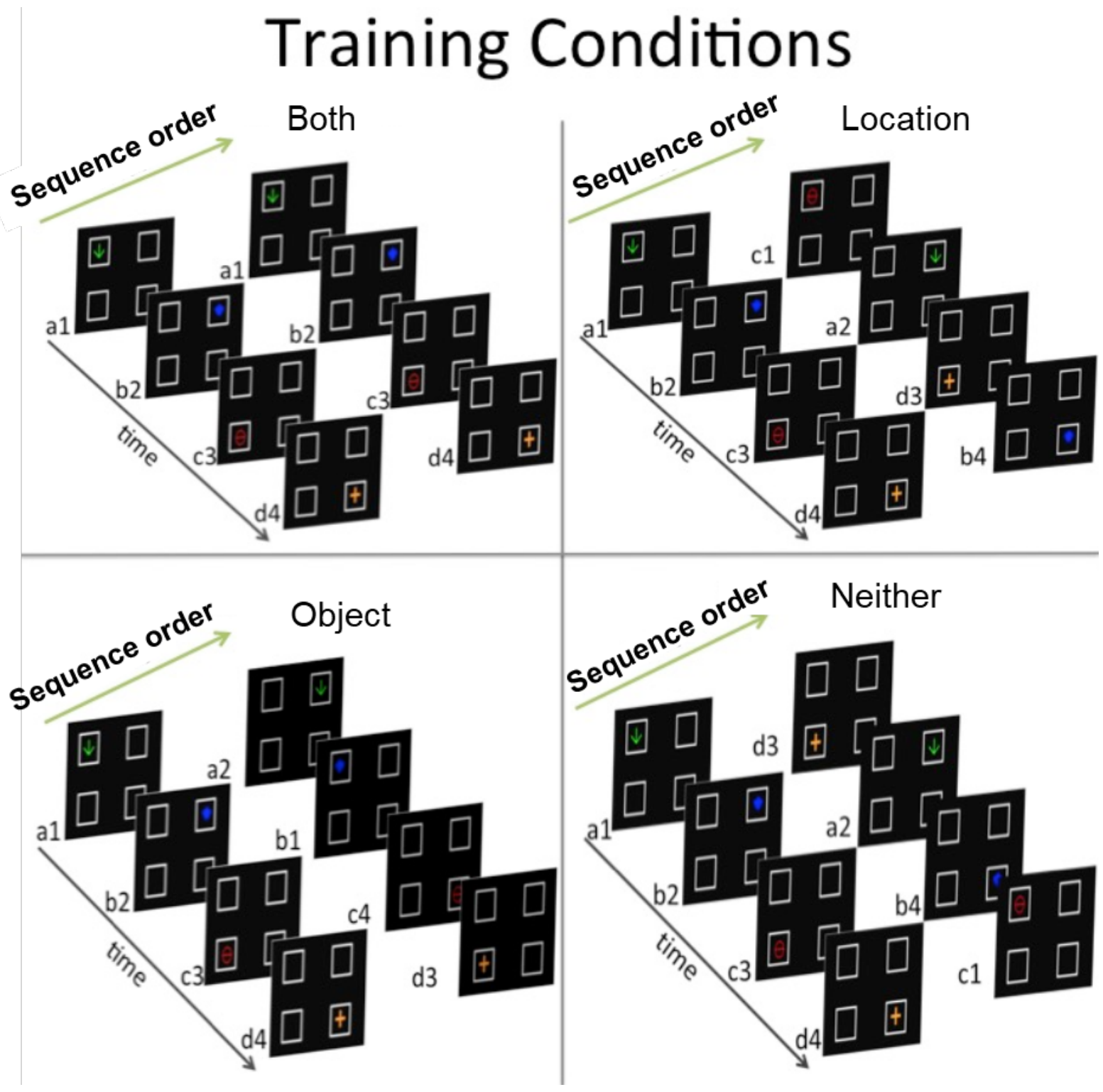

Figure 2. Examples of sequences used in training conditions in Experiment 1. Two consecutive 4-item sequences are shown for Group Both Training (top left), Location-Only Training (top right), Object-Only Training (bottom left), and Random Training (bottom right). 


\section{Probe Sequence Types}

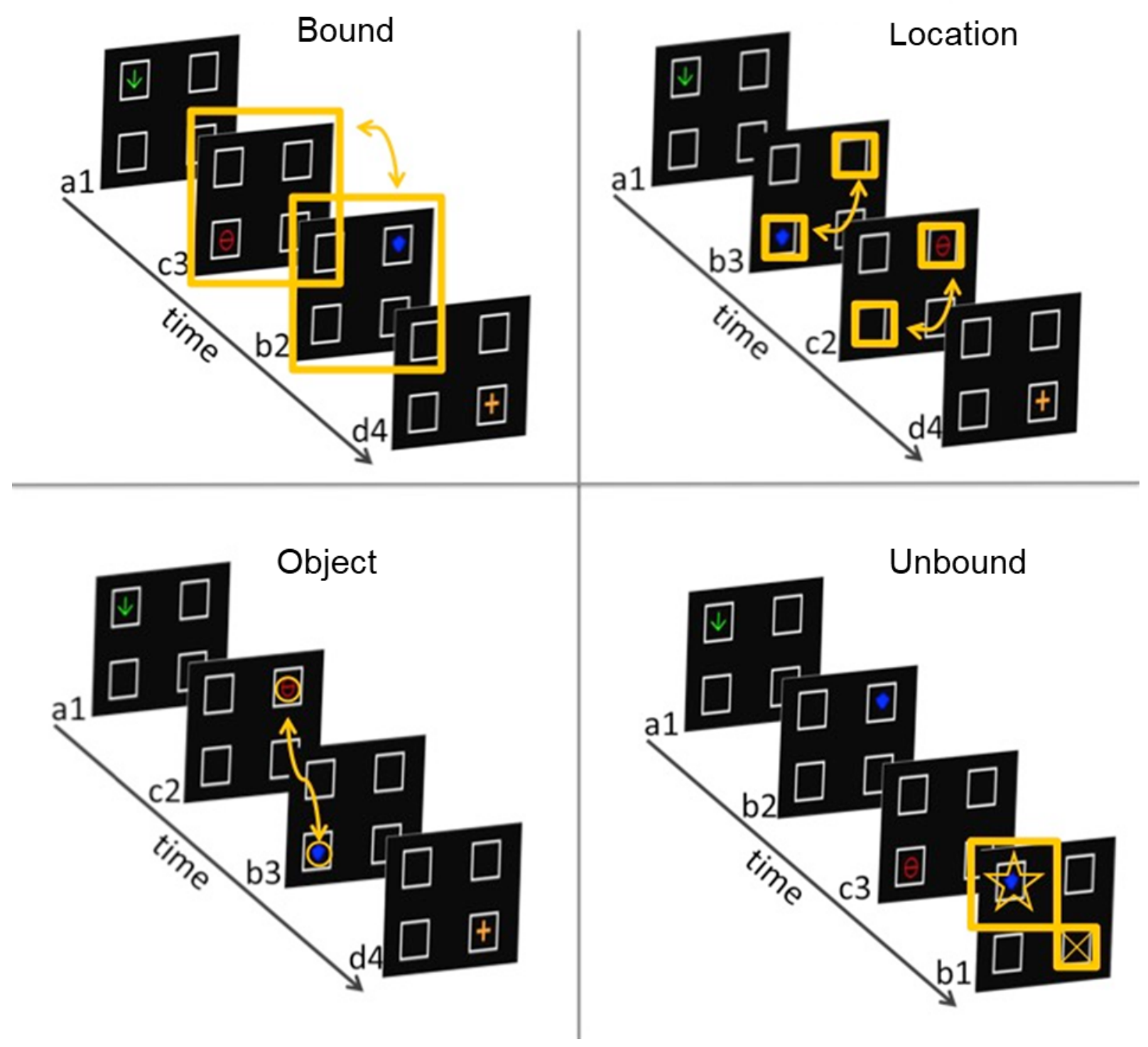

Figure 3. Examples of probe sequences used in testing in Experiments 1 and 2. In Bound probe sequences (top left), the order of two of the displays were swapped. In Location probe sequences (top right), the order of two locations were switched. In Object probe sequences (bottom left), the order of two of the objects were switched. In Unbound probe sequences (lower right), one of the items in the sequence consisted of a repeat of an object and location already used in that sequence such that the object-location association differed from that used in the training sequence. 


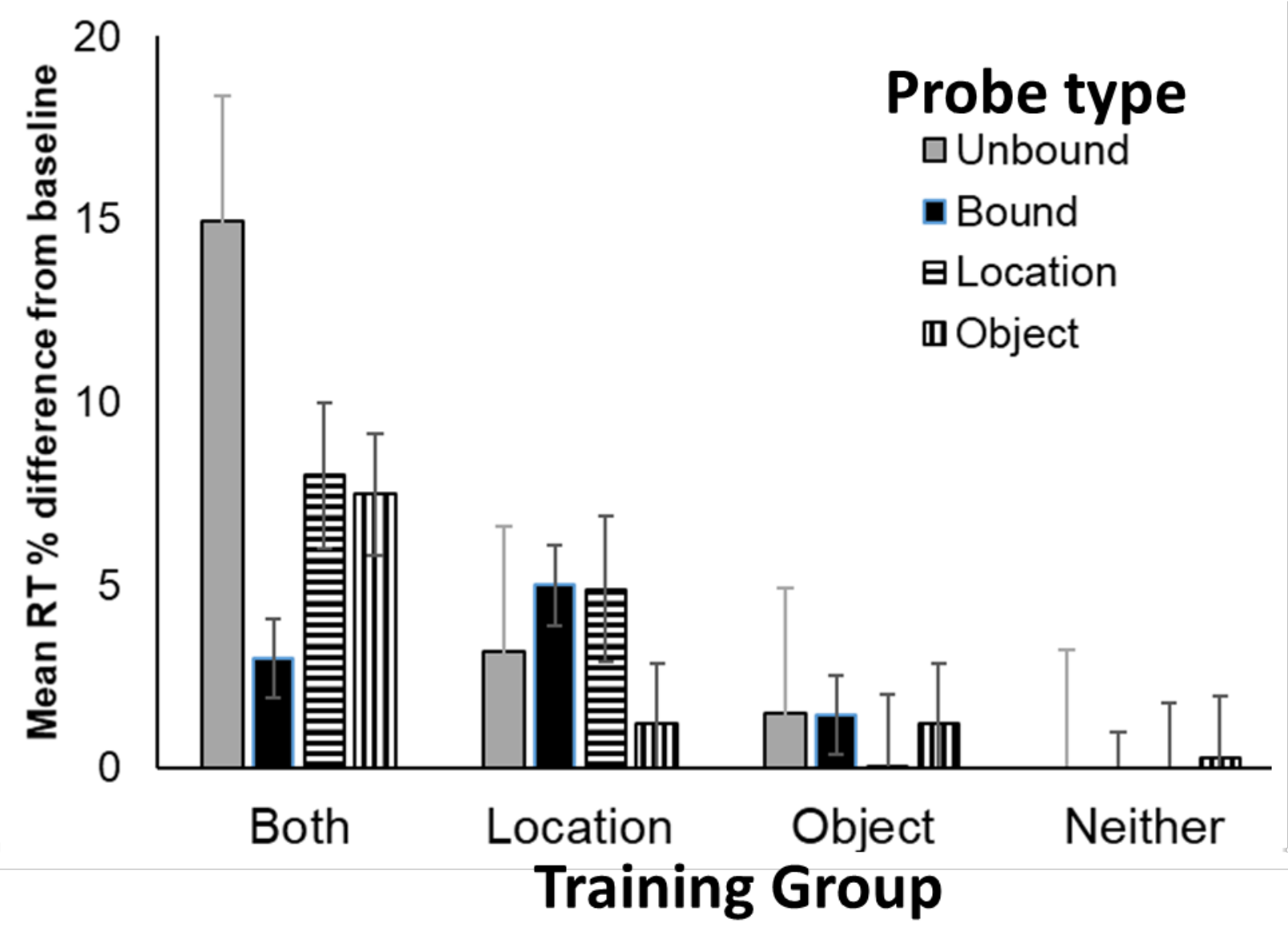

Figure 4. Mean Response Time (RT) cost in seconds for each probe sequence type (Unbound, Bound, Location, and Object) shown separately for Groups Both Training, Location-Only Training, Object-Only Training, and Random Training in Experiment 1. Error bars depict mean standard errors. 

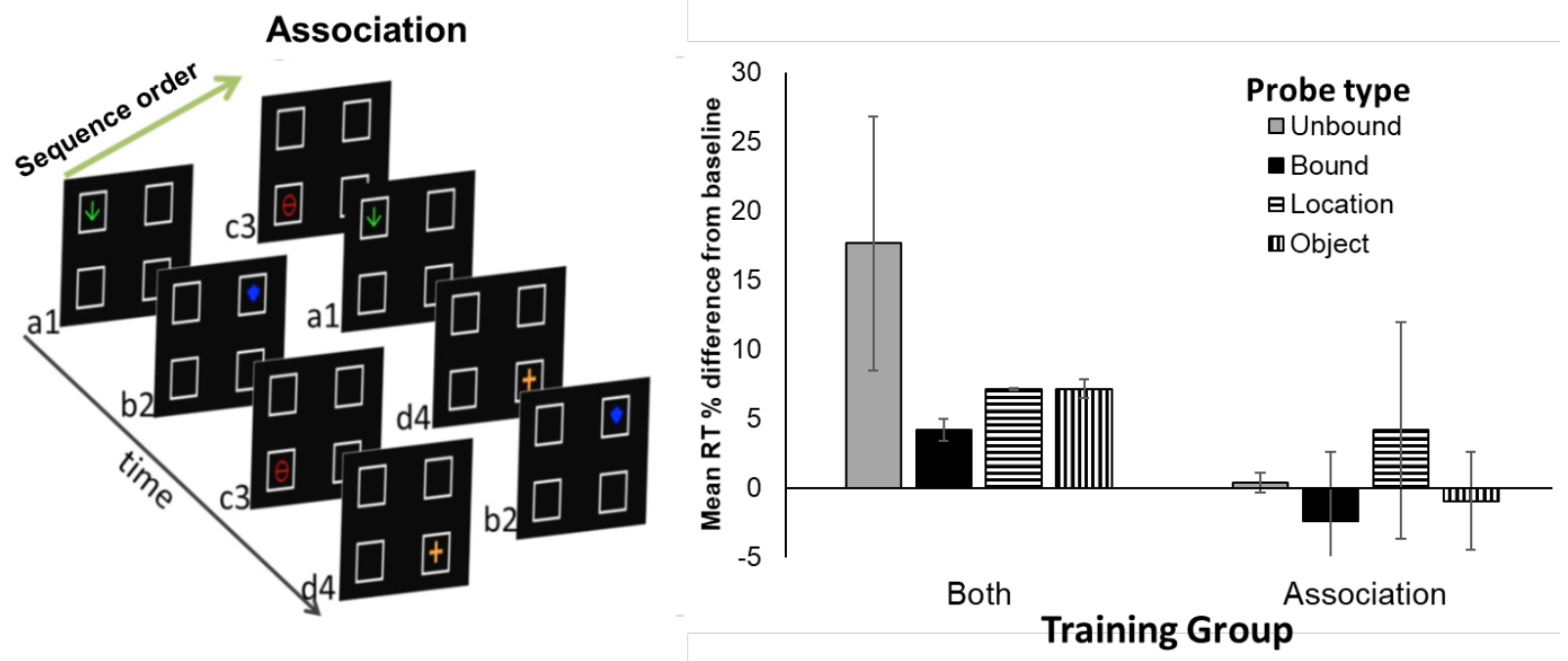

Figure 5. Left panel: Examples of sequences used in training condition for Group Association in Experiment 2. Right panel: Mean Response Time (RT) cost in seconds for each probe sequence type (Unbound, Bound, Location, and Object) shown separately for Groups Both Training and Association in Experiment 2. Error bars depict mean standard errors. 